\title{
Resonance Imaging in Hospital Work System Layout
}

\section{Conference Paper}

Author(s):

Held, Jürgen (1); Krueger, Helmut

Publication date:

2000-07

Permanent link:

https://doi.org/10.3929/ethz-b-000300421

\section{Rights / license:}

In Copyright - Non-Commercial Use Permitted

Originally published in:

Proceedings of the Human Factors and Ergonomics Society Annual Meeting 44(33), https://doi.org/10.1177/154193120004403343 


\title{
RESONANCE IMAGING \\ IN \\ HOSPITAL WORK SYSTEM LAYOUT ${ }^{1}$
}

\author{
Jürgen Held and Helmut Krueger \\ Institute of Hygiene and Applied Physiology \\ Swiss Federal Institute of Technology Zurich, Switzerland
}

\begin{abstract}
Context is the layout of a new multidiscipline facility with approx. 25 operating rooms (OR's). Focus lies on the question whether to work with rooms for preparation/induction of the patient or to do these procedures in the OR. Method is the analysis of work procedures and expert-user interviews in the university hospitals in Switzerland (Zurich, Bern, Lausanne), Belgium (Leuven) and USA (MAYO-Medical Center/Rochester). Task analysis is done with the FITSystem. The interviews are carried out with the VALAMO (variable layout model). The user's statements are structured in a database called MEDINO (management, ergonomics and design information for organisations). In contrast to the Swiss hospitals (6-8 OR's, induction rooms, no central holding areas), the MAYO-Clinic (45 OR's) as well as the hospital in Leuven (16 OR's) have central holding areas, an integrated post anaesthesia care unit. Both hospitals have induction rooms which are no longer in use due to cost constraints. The hospital in Leuven shows an efficient work in small teams without nurse anaesthetists and an OR layout which supports overview and supervision.
\end{abstract}

\section{INTRODUCTION}

\section{Resonance Imaging}

Resonance is useful to reach:

- an insight (magnetic resonance imaging)

- an harmonic tuning (music instruments)

- and a better understanding (communication)

In this work, resonance imaging is a metaphor for a process of information and knowledge transfer under the objectives of prospective ergonomics.

Context is the new design of an hospital building as part of the University Hospital of Bern (1000 beds) in /Switzerland (figure 1).

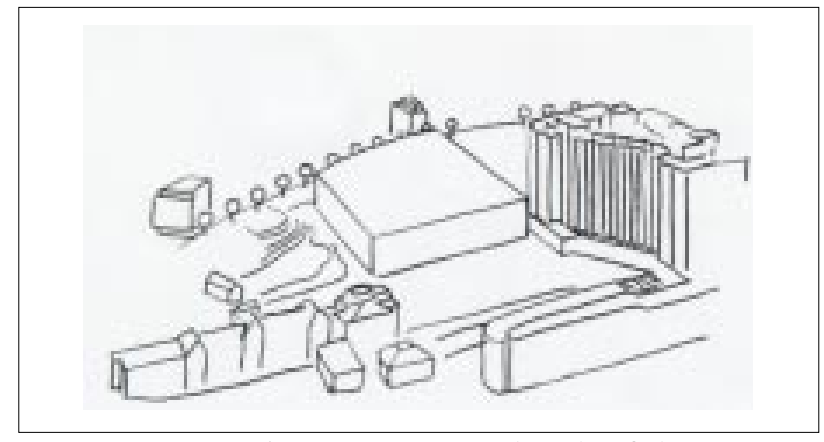

Figure 1: $\quad$ Sketch of the building's outline (block in the midst, length $280 \mathrm{ft}$., width $320 \mathrm{ft} ., 6$ stocks).
The project's name is INO. The abbreviation derives from the functional contents of the building, it includes:

- intensive care unit (Intensivbehandlung)

- emergency facility Notfallstation)

- surgical facility (Operationszentrum)

Completion is planned for the year 2005. Especially the surgical facility will cause an immense system change for the entire hospital. The situation nowadays shows 28 operating rooms (OR) at eight different locations on the campus. But the new INObuilding will have $25 \mathrm{OR}$ at one floor!

\section{What is the best layout?}

The INO-project provides an extensive usercentred approach to incorporate user knowledge in the design process. Multiple professions, an high amount of work processes and the work system's complexity leads naturally to diversity in user interests and requirements.

Especially in the discussion of the future OR facility layout. Hereby one question starts a controversy:

„Do we need induction rooms?“

\footnotetext{
${ }^{1}$ In: Proceedings of the IEA 2000/HFES 2000 Congress, Vol. 6, pp. 237 - 240.
} 
Induction means the tasks to narcotise the patient. In simple terms: Before induction the patient is awake but under medications (sedatives), after the induction he is narcotised.

The discussion is about two concepts:

a) The planning of a central holding area integrated in the surgical facility. The holding area is for patient waiting. Rooms (induction rooms) are linked to the OR's for patient induction/preparation (Concept A).

b) An holding area like in concept $\mathrm{A}$, but the idea is to do some preparations in advance in the holding area. There are no induction rooms. Induction and all other procedures are performed in the OR (Concept B).

Each concept will work with a central unit for post anaesthesia care.

The project management decided to clarify advantages and disadvantages of the both concepts. The intention is to take profit from user's knowledge in facilities, similarly to those described above.

The problem is to acquire and transfer such knowledge, characterise by the fact that: „We can know more than we can tell" (Polyani, 1967).

\section{METHOD}

Objects of analysis are the surgical facilities of the following five University Hospitals

1. University Hospital of Bern

2. University Hospital of Zurich

3. University Hospital of Lausanne

4. University Hospital Gasthuisberg in Leuven/Belgium

5. Mayo-Medical Center in Rochester/USA Minnesota

The analyses includes:

- literature research

- guided site visit

- observation of work procedures

- FIT-System analysis (Held \& Krueger 1999)

- semi-structured OR-user interview

The interviews were carried out with the VALAMO (variable layout model), an instrument to facilitate knowledge verbalisation. It's a mobile tool of magnetic objects on a metal work surface white, and a video camera system (Held \& Krueger, 1999).
The magnetic objects have the scale 1:100. They represent personal and equipment on different ground plans of surgical facilities.

All user statements are recorded with the video system of the VALAMO. Their transcription is structured under 19 topics:

1. Patient's safety

2. Patient's care

3. Patient's comfort

4. Patient's load of anaesthetic drugs

5. Turn over time

6. Preparation of the OR

7. Organisation of the OR-Staff

8. Team performance

9. Training

10. Ambulant surgery

11. Anaesthesia equipment

12. Ratio: amount of OR's for A/OR's for B

13. Holding Area

14. Post Anaesthesia Care Unit (PACU)

15. OR's for multiple and parallel surgery

16. Central area for patient's induction

17. Flexible and moveable walls in the OR

18. Combination of concept A andconcept B

19. Other concepts, ideas

The statements are structured in a data base: MEDINO, Management, Ergonomics and Design Information for Organisations.

\section{RESULTS}

\section{The Dispute in Literature}

Few publications exists to the question of induction rooms for surgical facilities. Friesdorf (1984) propose to install induction rooms outside of the OR facility. This under the aspect to provide a calm and friendly atmosphere. Holst and Wendt (1996) want to divide the OR in two rooms. The intention is a simultaneously patient induction and preparation and surgery to optimise the work process and to reduce personal costs. Without induction rooms, the patient is affronted with the cold, anxious and noisy atmosphere of the OR. Some authors argue, that hygiene can better controlled when each OR has induction rooms. 


\section{The Five Hospitals}

The different surgical facilities have the following characteristics:

1. University Hospital of Bern

Erected 1964; 6 OR's on one floor; surface of one OR: 495 sq. ft.; induction rooms for each OR; no holding area.

2. University Hospital of Zurich

Redesigned 1990; 8 OR's on one floor; surface of one OR: 420 sq. ft.; induction rooms for each OR; no holding area.

3. University Hospital of Lausanne

Erected 1980; 18 OR's on one floor; surface of one OR: 387 sq. ft.; induction rooms for each OR

- but no longer in use; small holding area combined with a post anaesthesia care unit.

4. University Hospitel Gasthuisberg/Leuven Erected 1985; 16 OR's on one floor; surface of one OR: 452 sq. ft.; enlarged 1998 to 22 OR's; one induction room for two OR's - but no longer in use; holding area; post anaesthesia care unit.

5. MAYO Clinic: Saint Marys Hospital

Erected 1981; 53 OR's on one floor; 43 in use, surface of one OR: 360 sq. ft.; some induction rooms - but no longer in use; holding area; post anaesthesia care unit.

The layout of the facilities shows three different structures:

\section{a) Single Row}

Due to the small amount of OR's Bern and Zurich have a single row layout. The OR's stand side by the side, the induction rooms are in front of it (figure 1).

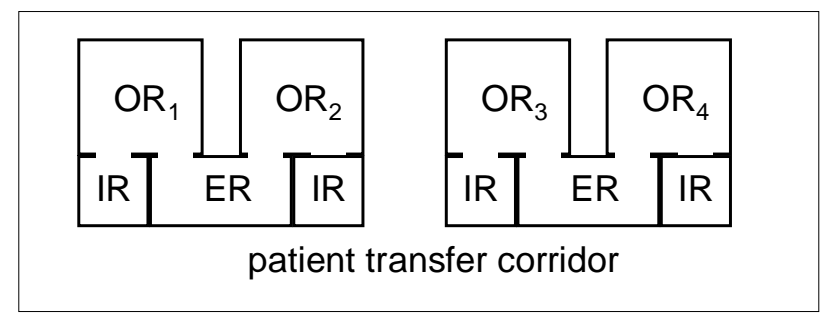

Figure 1: Example of a row of four OR's, induction rooms (IR) and rooms for extubation (ER). b) Parallel Rows

Lausannne and the MAYO-Clinic have a structure of parallel rows. In Lausanne the induction rooms are in front of the OR's, at the MAYO Clinic between two OR's (figure 2).

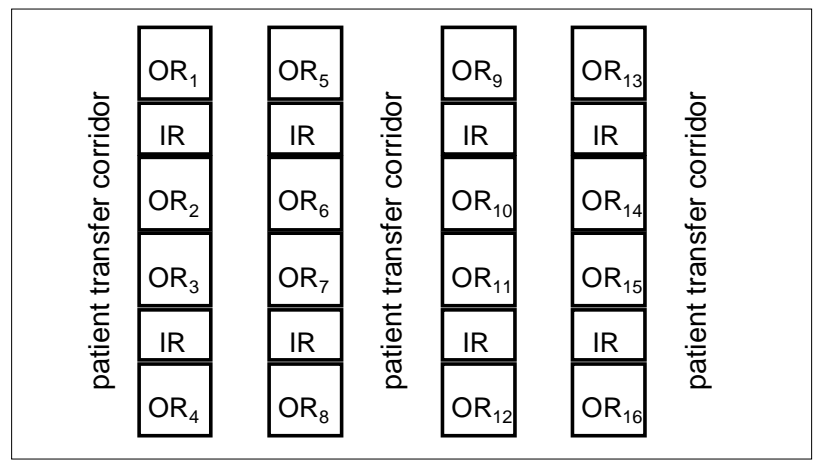

Figure 2: Parallel row layout of OR's, the induction rooms (IR) are between two OR's.

c) Clusters

The facility in Leuven has a structure of four clusters. Each cluster contents four OR's (figure 3).

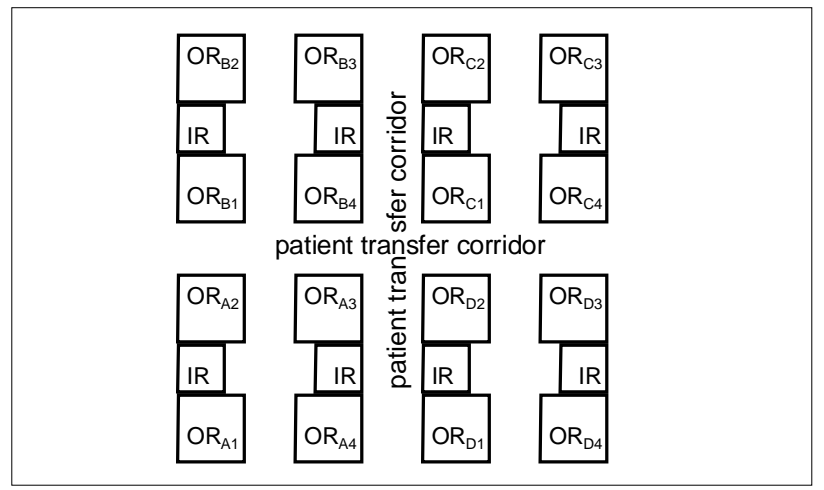

Figure 3: Clusters of four OR's, the induction rooms (IR) are between two OR's.

\section{The User's Voices}

The interview resulted in 597 statements from 41 users (Anaesthetists, Surgeons, Nurse Anaesthetists, OR-Nurses, Specialists) related to the both concepts (A: Induction rooms, B: No induction rooms). Video documentation of the VALAMO sessions show the various work procedures and user explications. The FIT-System analysis shows the distribution of tasks to team members during patient preparation (see figure 4 on the next page).

The following list of aspects give a summarised insight in the information base: 
- Patient's safety - No problems (hygiene, infections) were mentioned in both concepts.

- Patient's care - The constant relation between one and the same anaesthetist and the patient is of importance. But isn't practicable in concept B.

- Patient's comfort - The users of concept B reduce all noise until the patient's intubation is over.

- Turn over time - The extubation is a uncertainly time factor and can block the OR.

- Preparation of the OR - In the Swiss hospitals it isn't allowed to enter the OR until the set-up of the instruments are finished.

- Organisation of the OR-Staff - Personal cost are reduced in Leuven by team work (they have no nurse anaesthetists) and by supervision (one senior anaesthetist for four OR's).

- Ambulant surgery: - OR's are blocked by the high amount of walk in patients in the morning hour (hospital in Leuven).

- Ratio: amount of OR's for A/OR's for B - In concept $\mathrm{B}$, the need is $25 \%$ more OR's to cope with the same amount of surgical procedure.

- Holding Area - Lack of patient's privacy and orientation for the physician causes problems (Leuven, MAYO).

- Post Anesthesia Care Unit (PACU) - A bottleneck (The hospital in Leuven must enlarge their PACU), requires separation according to the patients (children, ambulant/stationary).

\section{DISCUSSION AND CONCLUSION}

Most architects never worked in surgical facilities. Therefore it's a matter of course to incorporate users knowledge in hospital work system layout. The problems hereby are the acquisition and transfer of knowledge. Natural users are not aware of their daily work. „Preparation is a block of $\mathrm{x}$ minutes“ is the user's statement - but in fact is this divers allocated to team members and small sub tasks (figure 4). Tools like the FIT-System can easily inspect work and can effect confrontation and process awareness at the user's side. Further the VALAMO facilitates knowledge transfer by „,naming-cum-pointing“ (Polyani, 1967). Those user-ergonomist interactions supports mutual learning processes. But than mutual learning is still the challenge in interactions between ergonomist and system planers. After all, layout discussions can not distract, that everything depends on the staffing.
Whatever the experts in architecture and hospital management will decide about the building, the quality of expert-user interactions will decide about acceptance and success.

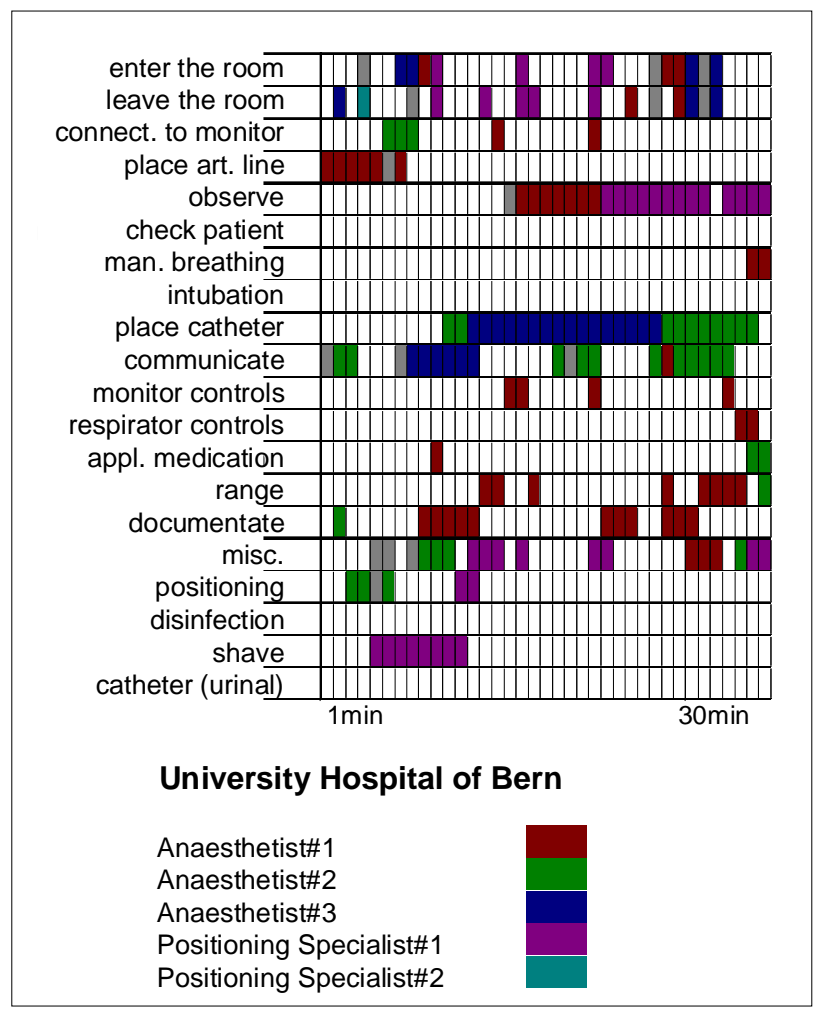

Figure 4: FIT-System analysis of the tasks for preparation/induction of the patient.

\section{REFERENCES}

Held, J. \& Krueger, H. (2000). The Ergonomic Observer - A new flexible Interface Technique for online Work and Teamwork analysis. In Proc. XIVth Triennial Congress of the International Ergonomics Association. San Diego 2000.

Held, J. and Krueger, H. (1999). The Ice-breaking VALAMO - A Tool for Participatory Processes In Proc. of the $8^{\text {th }}$ Int. Conference on HumanComputer Interaction (pp. 563-567), Munich. Polanyi, M. (1967). The Tacit Dimension. London: Routledge \& Kegan.

Friesdorf, W. (Eds.) (1984). PAMS - PatientAnästhesist-Maschine - System, Zentrum für Anästhesiology, University Hospital Ulm.

Holst, D. \& Wendt, M. (1996). Ist unsere OPKonzeption heute noch zeitgerecht? Neue Ablaufkonzepte in der Anästhesie.

Anästhesiologie \& Intensivmedizin, 6, 315-319. 